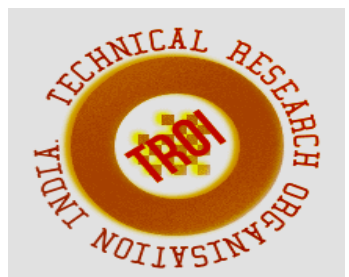

\title{
HIGH SPEED AND LOW AREA FIR FILTER IMPLEMENTATION BASED ON SHIFT AND ADD MULTIPLIER DESIGN FOR MACHINE LEARNING APPLICATIONS
}

\author{
${ }^{\# 1}$ Prof.Ruckmani Divakaran, ${ }^{\# 2}$ Arshiya Taj A., ${ }^{\# 3}$ Nikhath Suman, ${ }^{\# 4}$ Sneha S., ${ }^{\# 5}$ Sweety Lydia B. \\ ${ }^{1}$ Dept of Electronics and Communication Engineering, Dr.T.Thimmaiah Institute of Technology, \\ Visvesvaraya Technological University, Belagavi, Karnataka, India \\ hod.ece@drttit.edu.in \\ ${ }^{2}$ Dept of Electronics and Communication Engineering, Dr.T.Thimmaiah Institute of Technology, \\ Visvesvaraya Technological university, Belagavi, Karnataka, India \\ snehashiva97@gmail.com
}

\begin{abstract}
Today every circuit has to face the power consumption issue for both portable device aiming at large battery life and high end circuits avoiding cooling packages and reliability issues that are too complex. It is generally accepted that during logic synthesis power tracks which works well with area.
\end{abstract} This means that a larger design will generally consume more power. The multiplier is an important kernel of digital signal processors. Because of the circuit complexity, the power consumption and area are the two important design considerations of the multiplier. In this paper a High Speed \& low area architecture for the shift and add multiplier is proposed. The simulation result for 8 bit multipliers \& four tap Filters shows that the proposed Low Area \& Delay architecture lowers the total Area \& Delay when compared to the Array Multiplier and Booth Multiplier architecture based Filter. To develop the system blocks in Modelsim 6.4a and Xilinx ISE9.1i, the Spartan3 FPGA tool is used which achieves the simulation and the synthesis of the proposed multiplier. Verilog HDL is the language used for designing the proposed multiplier. Index Terms: Finite Impulse Response(FIR), Shift and Add Multiplier

\section{INTRODUCTION}

Shift-and-add multiplication is similar to the multiplication performed manually. The method adds the multiplicand ' $A$ ' to itself ' $B$ ' times where ' $B$ ' denotes the multiplier. To perform the entire multiplication for getting the final product, the conventional architecture that was used for shift and add multipliers required many switching activities. So the power dissipation was more in that. By removing the sources switching activity in the older multiplier, low power architecture of multiplier can be achieved.

This also reduces the energy consumption of the accelerator which satisfies the requirements compared to the previous multipliers. Various different fixed-coefficient multipliers were proposed in the DSP domain[5],it used lookup table but in this proposed multiplier lookup table is not used

\section{NEURAL NETWORK:}

Recent study on Neural Network (NN) was showing good advancement over the previous algorithms in machine learning. Different network models, like recurrent neural network (RNN), and convolutional neural network (CNN), have been proposed for video ,image \& speech process. Artificial Neural Network. ANNs were usually presented as the systems shown in the below Figure 1 is of interconnected nodes called neurons. There are a many articles on architecture for neural acceleration.[1],[2],[3]-[4]. Each and every neuron generates the single 
output by operating on the vector of inputs, represented by $\mathrm{x}$ here. The input vector was associated with the weight vector (represented by w), to identify every entry's numerical significance. Here in the mathematical representation, the neurons will first have to compute a weighted sum, and then they will perform a non-linear activation function on the weighted sum in order to generate the output $(x)$.

$$
\begin{gathered}
g(x)=f\left(\sum_{i=1}^{d} x_{i} w_{i}+w_{0}\right)=f\left(w^{t} x\right) \\
f(s)=\left\{\begin{array}{c}
1, \text { if } s \geq 0 \\
-1, \text { if } s<0
\end{array}\right.
\end{gathered}
$$

Usually, an artificial neural network is defined by three parameters :

1. Interconnection pattern between the different layers of the neurons.

2. The activation function that helps in converting a neuron's weighted input to its output activation;

3. The learning process in updating the weights of the interconnections.

The Error tolerance of the Neural Network (NN) makes appropriate computing the promising technique in order to improve the energy efficiency of neural network inference. Earlier approximate computing focused on balancing the trade-off efficiency-accuracy for the existing pre-trained networks, which would lead to suboptimal solutions.

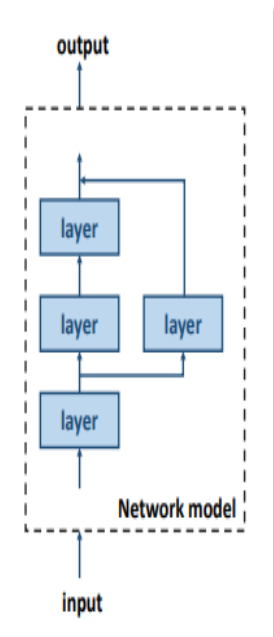

(a)

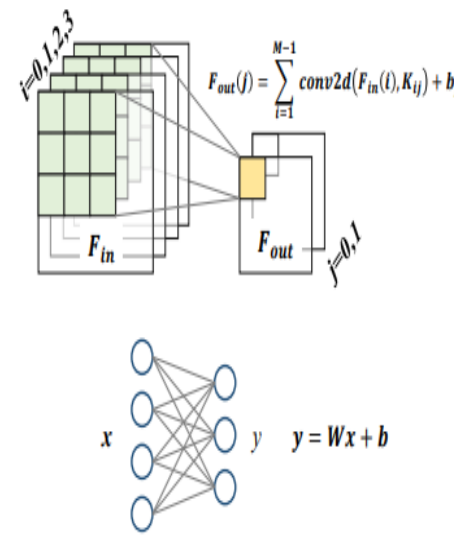

(b)
Figure1.Neural Network Model

In this paper the neural network network is considered because it is most widely used in machine learning. The designed multiplier will act as the key hardware component to the neural network. Hence the neural network is implemented using this multilplier and the results obtained are seen.Figure 1 represents the basic general neural network model and the below Figure2 represents the fuzzy storage model of the network including neurons which does processing.

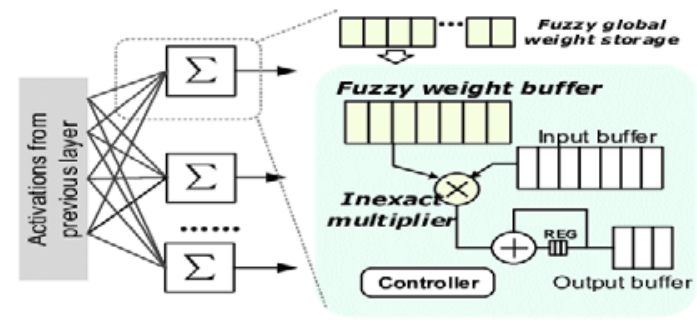

Figure 2. Fuzzy storage model

\section{II.METHODOLOGY}

The Architecture of the Simul Multiplier as shown in the below diagram consist of the following components.

Shift and Add Multiplier

Registers

Adder

Shifter

Counter 


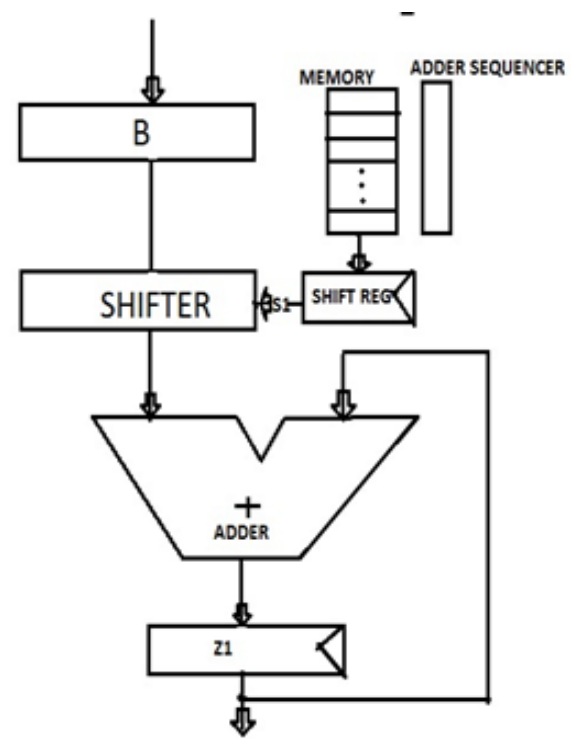

Figure 3.Simul Multiplier Block Diagram

SIMUL is an (Significant - Driven Iterative Approximate Multiplier) as shown in the above Figure 3 is an approximate multiplier features uses control precision that exploits the common characteristics ML Algorithm and it also supports a trade off between the compute precision and energy consumption at run time. Kulkarni et al[17] and Gupta et al[18] they worked together and proposed under-designed multiplier and adder designs in which the minimum and the maximum precision is being fixed at the design time. In the year 2011 Babica et al [11] they have been proposed an ILAM(Iterative Logarithmic multiplier) that can support different precision through the iterations, but our proposed shift-and-add Multiplier is far more energy-efficient with a smaller area than the ILAM. Proposed In the year In the year 1996 K.Chapman [19] proposed a 16 12-bit entries for an 8x8 multiplier per coefficient but our proposed design requires only one 20-bit entry per coefficient.Shift-and-add multiplication is same as the multiplication performed manually by the human. This method adds the ' $\mathrm{B}$ ' denotes the multiplier. To multiply two numbers by multiplicand ' $A$ ' to itself ' $B$ ' times, which is performed in a paper, this method takes the digits of the multiplicand by a single digit of the intermediate product in the appropriate positions to the left of the earlier results. To perform the complete operations for getting the final product, the conventional architecture for the shift and add multipliers require many switching activities. In the conventional architecture there is more dissipation of the dynamic power. In this conventional multiplier by eliminating or reducing the sources switching activity, the low power architecture of multiplier can be derived. This is one among digital system of the functional component, the reduction of power dissipation in multipliers should be as much as possible.

\section{TESTS AND RESULTS}

The proposed multiplier is based on multiplicands, multiplier, coefficient memory, shift register and adder which is simulated by providing reset, clock and inputs using Modelsim 6.4a and simulation results as shown in the below Figure 4.

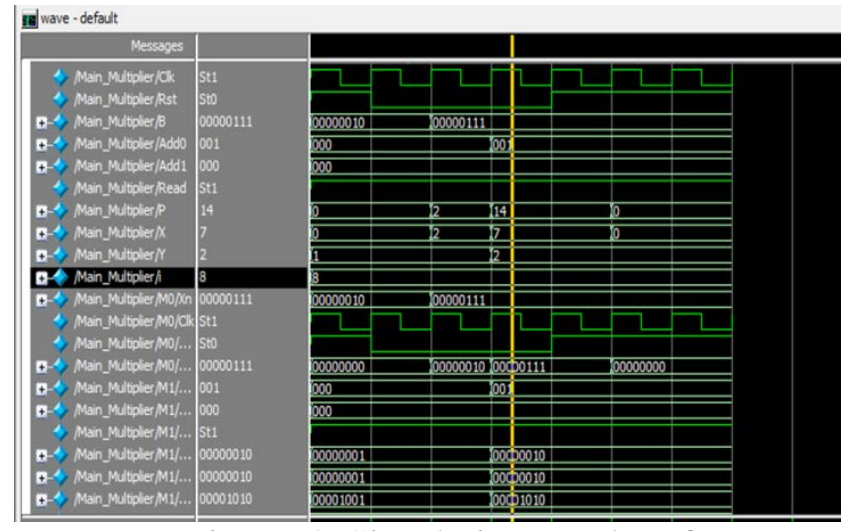

Figure 4. Simulation results of multiplier

The proposed multiplier is synthesized using Xilinx Synthesis Tool (XST) and Xilinx ISE v9.1i as the development environment. The RTL schematic of the overall design is shown in below Figure 5.

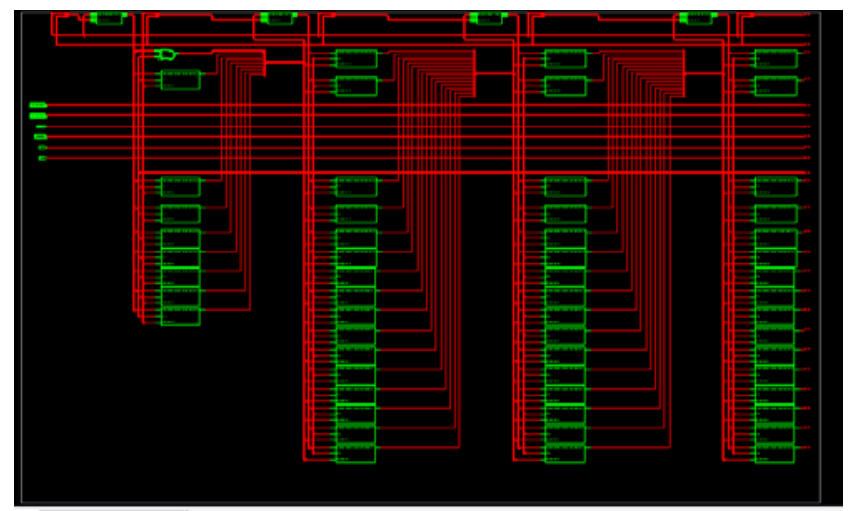

Figure 5. RTL Schematic of Simul Multiplier 
The device utilization summary of Shift-andAdd multiplier ,booth and the array multiplier is shown in below Figure 6,Figure 7,and Figure 8.

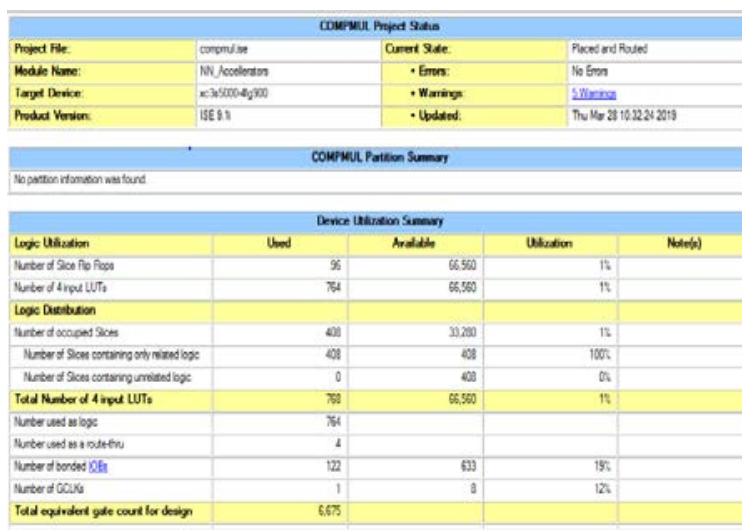

Figure 6. device utilization summary architecture of Shift-and-Add multiplier

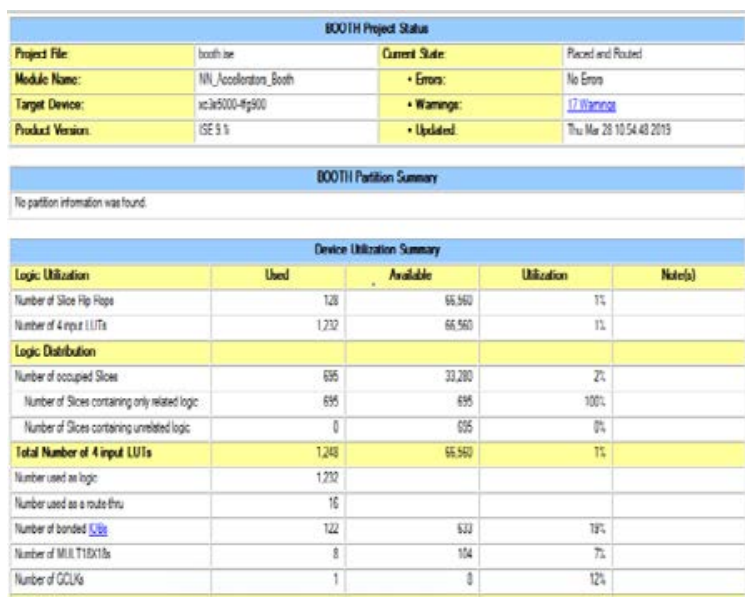

Figure 7 device utilization summary architecture of Booth Multiplier

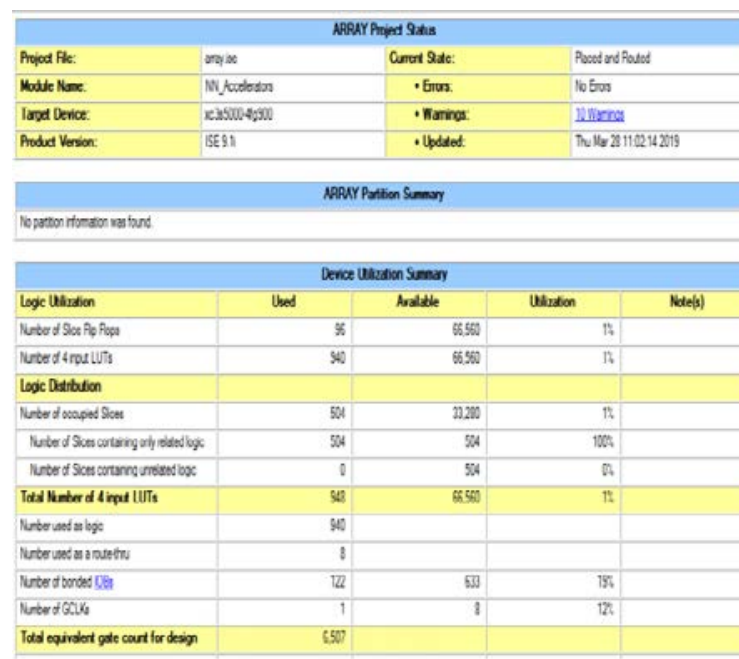

Figure 8.Device utilization summary architecture of Array Multiplier

The proposed multiplier is designed as the application part in the Neural Acceleration which can be used in the machine learning as shown in below Figure 9.

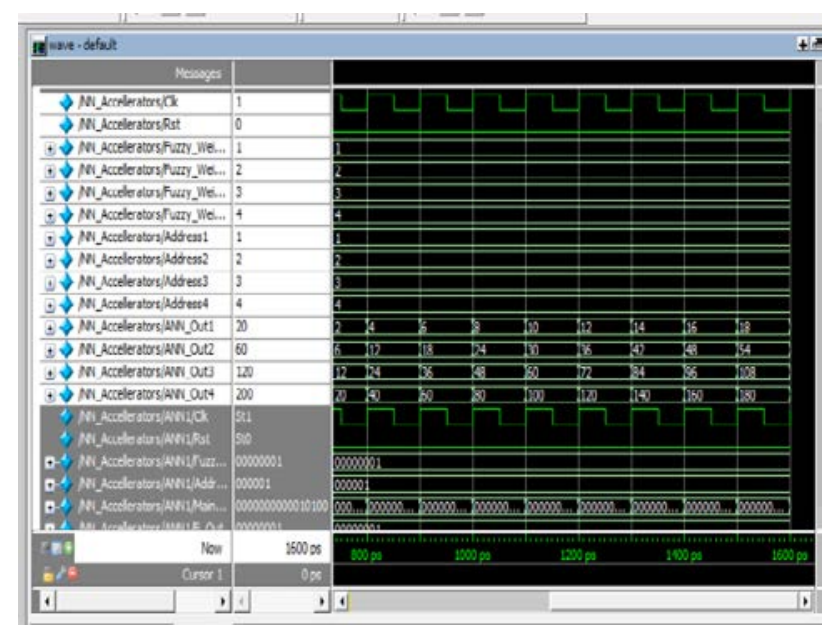

Figure 9. Simulation result of Neural accelerator

\begin{tabular}{|c|c|c|c|c|}
\hline $\begin{array}{c}\text { Device } \\
\text { name }\end{array}$ & \multicolumn{3}{|c|}{ Area } & Delay \\
\hline $\begin{array}{c}\text { FPGA } \\
\text { Spartan3XC } \\
\text { 3S5000 } \\
\text { FG 900-4 }\end{array}$ & $\begin{array}{c}\text { Slice } \\
\text { s }\end{array}$ & $\begin{array}{c}\text { LU } \\
\text { T }\end{array}$ & $\begin{array}{c}\text { Gat } \\
\text { e }\end{array}$ & Delay \\
\hline $\begin{array}{c}\text { Booth } \\
\text { Multiplier }\end{array}$ & 695 & $\begin{array}{c}124 \\
8\end{array}$ & $\begin{array}{c}4197 \\
9\end{array}$ & $\begin{array}{c}11.162 \\
\text { ns }\end{array}$ \\
\hline $\begin{array}{c}\text { Array } \\
\text { Multiplier }\end{array}$ & 504 & 948 & 6507 & $\begin{array}{c}10.979 \\
\text { ns }\end{array}$ \\
\hline $\begin{array}{c}\text { Shift and } \\
\text { Add } \\
\text { Multiplier }\end{array}$ & 408 & 768 & 6675 & 10.835 \\
ns
\end{tabular}

Figure 10 Comparison Table

The Comparison table and the graphical representation of the Shift-and-add multiplier along with the booth and the array multiplier is shown in the below Figure 10, Figure 11 and Figure 12. 


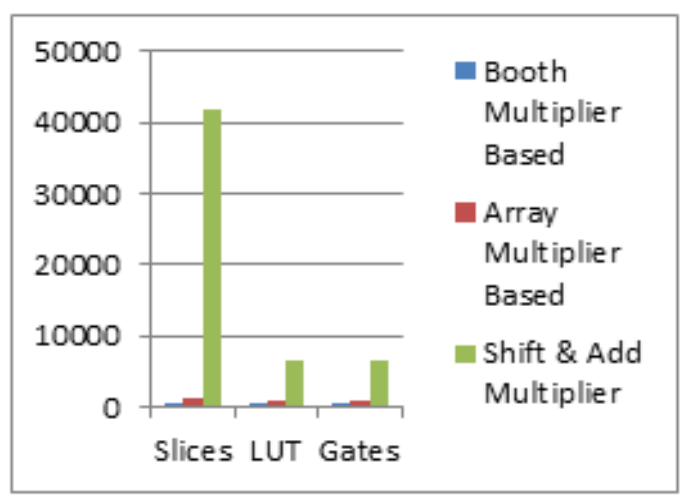

Figure 11. Area Comparision Graph

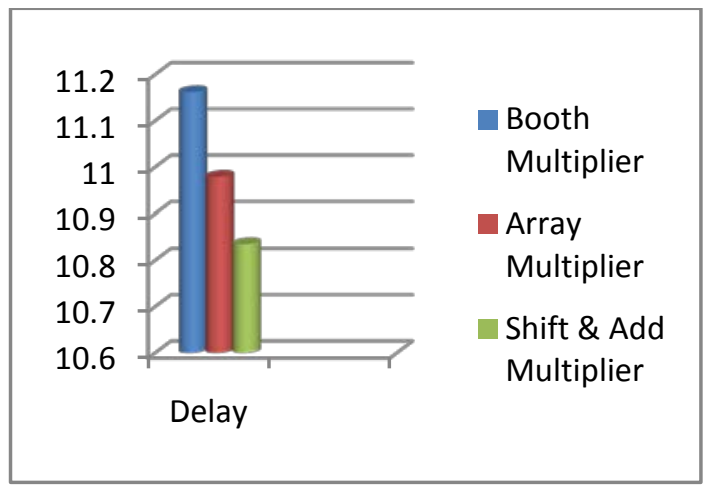

Figure 12. Delay Comparision Graph

\section{CONCLUSION}

The proposed architecture Area and Delay Efficiency when compared to a Booth Multiplier and Array Multiplier the proposed architecture makes use of bit width control logic and a low Area and Delay. The simulation result for 8 bit multipliers \& four tap Filters shows that the proposed low Area and Delay architecture lowers the total Area \& Delay when compared to the Array Multiplier and Booth Multiplier architecture based Filter. The design can be verified using Modelsim 6.4a with Verilog HDL code and Area and Delay is analyzed using Xilinx software. From Table and Comparison Graph, proposed architecture can attain less area and less Delay when compared to the conventional Array Multiplier and Booth multipliers.

\section{ACKNOWLEDGEMENT}

We would like to express our sincere gratitude to our Guide, HOD and Dean Administration Prof. Ruckmani Divakaran for her constant encouragement and excellent guidance without which we wouldn't have accomplished this. We wish to express our hearty thanks to our Principal Dr. Syed Ariff for providing good infrastructure for undergoing this work in college. We would like to thank all faculty members and staffs who were responsible for carrying out this project work successfully. We extend our hearty thanks to our parents and friends for all the moral support provided during the presentation for the project work.

\section{REFERENCES}

[1] H. Esmaeilzadeh et al., "Dark Silicon and the End of Multicore Scaling," Proceedings of the 38th International Symposium on Computer Architecture (ISCA), 2011.

[2] J. Park et al., "Scale-out Acceleration for Machine Learning," Proceedings of the 50th Annual IEEE/ACM

International Symposium on

Microarchitecture (MICRO), 2017.

[3] H. Sharman et al., "Bit Fusion: Bit-Level Dynamically Composable Architecture for Accelerating Deep Neural Networks," Proceedings of the 45th International Symposium on Computer Architecture, 2018.
[4] J. Albericio et al., "Bit-Pragmatic Deep Neural Network

Computing," Proceedings of the 50th

Annual IEEE/ACM International Symposium on Microarchitecture (MICRO), 2017.

[5] B. Reagen et al., "Minerva: Enabling Low- Power, Highly Accurate Deep Neural Network

Accelerators," Proceedings of the 43rd International Symposium on Computer Architecture (ISCA), 2016

[6] D. Verstraeten et al., "Isolated Word Recognition with the Liquid State Machine: A Case Study,” Information Processing Letters, September 2005.

[7] A. Damien, Tensor flow the Examples "Neural Networks/ multilayer”_perceptron.py.

[8] Y. LeCun et al., "The MNIST database of handwritten digits". [9] M. Liberman et al., "The TI 46word speech database".

[10] O. Sakhi, "Face Detection using Support Vector Machine(SVM)”.

[11] Z. Babić, A. Avramović, and P. Bulić, “An Iterative 
Logarithmic

Microprocessors and Microsystems, February 2011.

\section{Multiplier,"}

[12] H. Esmaeilzadeh et al., "Neural

Acceleration for General-Purpose

Approximate Programs,”

Proceedings of the 45th Annual

IEEE/ACM International Symposium

on Microarchitecture (MICRO), 2012.

[13] A. Yazdanbakhsh et al., “AxBench: A Multi- Platform

Benchmark Suite for Approximate

Computing," IEEE Design \& Test,April 2017.

[14] V. K. Chippa et al., "Scalable Effort Hardware Design: xploiting Algorithmic Resilience for Energy Efficiency," Proceedings of the 47th

Design Automation Conference (DAC), 2010.

[15] A. Yazdanbakhsh et al., “Neural Acceleration for GPU Throughput Processors," Proceedings of the 48th International Symposium on Microarchitecture, 2015.

[16] A Yazdanbakhsh et al., “GANAX:

A Unified SIMD-MIMD

Acceleration for Generative Adversarial Network," Proceedings of the 45th International Symposium on Computer Architecture, 2018. [17] P. Kulkarni, P. Gupta, and M. Ercegovac, “Trading Accuracy for Power with an Underdesigned Multiplier Architecture," Proceedings of the 24th International Conference on VLSI Design, 2011.

[18] V. Gupta et al., "IMPACT: IMPrecise adders for lowpower approximate computing," International Symposium on Low Power Electronics and Design (ISLPED), 2011.

[19] K. Chapman, "Constant Coefficient Multipliers for the XC4000E," 1996. 